Copyright (C) 2014 by Academic Publishing House Researcher

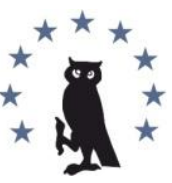

Published in the Russian Federation

European Researcher

Has been issued since 2010.

ISSN 2219-8229

E-ISSN 2224-0136

Vol. 84, No. 10-1, pp. 1776-1781, 2014

DOI: 10.13187/er.2014.84.1776

www.erjournal.ru

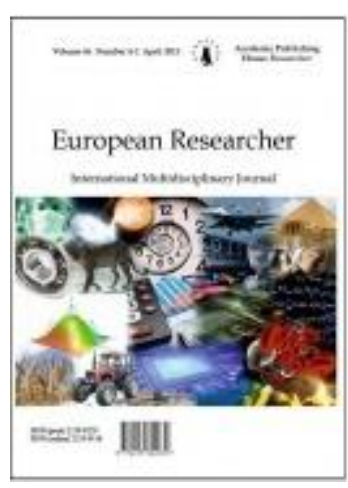

UDC 339.138

\title{
Development of Geo-Marketing
}

Tatiana Ozhereleva

Moscow State University of Geodesy and Cartography, Russian Federation

Senior Lecturer

E-mail: ozerotana@yandex.ru

\section{Abstract}

This article analyzes the state and development of geo-marketing. The author illustrates the multi-aspectedness of geo-marketing: applied technology and management technology. The article demonstrates that geo-marketing can be viewed as a reflection of the processes of co-evolution in society. The author brings to light the specifics of geo-marketing research and situational analysis in geo-marketing. The article describes applications of geo-marketing.

Keywords: marketing; geo-marketing; spatial economics; information technology; management information technology.

\section{Введение}

Как прикладная технология геомаркетинг является новой информационной технологией, сформировавшейся на основе интеграции технологий классического маркетинга и геоинформационных технологий. Как технология управления геомаркетинг возник как синтез технологий поддержки принятия решений, технологий визуальной обработки информации, маркетинговых технологий, интеграции различных данных и их комплексной обработки. В России довольно долго этому направлению не придавали внимания. За исключением работ профессора В.Я. Цветкова [1-5] первоначально исследованию геомаркетинга уделялось мало внимания. В настоящее время геомаркетинг значительно расширил сферу своего применения [6] и теоретическую основу, что делает актуальным проведение анализа его развития и применения.

\section{Основы появления и эволюция}

Причиной, обусловившей появление геомаркетинга, являются потребности бизнеса и муниципального управления [7]. В западной печати понятие геомаркетинг (geomarketing), связывает в неразрывное целое бизнес и геоинформационные технологии. За рубежом геомаркетинг в явной форме используется в туризме, логистике, при продаже геодезического обрудования, при предпроектных исследованиях, при продаже данных дистанционного зондирования, при инженерных и экологических изысканиях, при предпроектном обосновании строительства объектов сферы обслуживания, при размещении объектов сервиса и точек по оказанию государственных информационных услуг.

Первоначально геомаркетинг, как технология, возник на основе синтеза технологий обычного маркетинга, информационного маркетинга и геоинформационных технологий [5]. 
Концептуально его можно рассматривать и как концепцию рыночного ведения хозяйства. Однако далее его развитие осуществлялось в соответствии с принцип синергетики самоорганизацией, или коэволюцией [8]

На первых этапах применения геомаркетинг использовался и используется как средство деловой графики для качественного анализа и поддержки принятия решений. На следующем этапе появилась необходимость не только сбора, но и хранения данных. Возникла потребность использования баз данных для хранения поиска и организации запросов и электронных таблиц для проведения расчетов, оценок и анализа, включая финансовый. Далее появилась необходимость не только сбора и хранения, но и комплексной обработки данных, то есть возникла потребность интеграции как технологий, так и данных. Возникла задача согласования и самосогласования различных видов информации применяемой в геомаркетинге. Кроме того, возникла потребность использования других информационных источников, получаемых вне технологий маркетинга.

Решение этих задач стало возможным только на основе внутренней и внешней интеграции. Внутренняя интеграция основана на согласовании и оптимизации (коэволюции) форм и структур данных. В аспекте информационных технологий это называют структурной согласованностью и информационным соответствием [9].

Внешняя интеграция основана на коэволюции методов обработки маркетинга в геоинформационные технологии и геоинформатику. Поэтому, говоря в дальнейшем о методах обработки данных в геомаркетинге, необходимо иметь в виду их согласованность с методами геоинформатики. Геомаркетинг связывает маркетинг и синергетику. Дальнейшая коэволюция геомаркетинга осуществлялась на основе требований развития общества и в первую очередь требований новой экономики [10].

В итоге, возникнув как технология, геомаркетинг, в соответствии с требованиями информатизации и экономической информатики, превратился в новое направление, универсальный инструмент анализа и управления, выходящий далеко за рамки области маркетинга.

Как следствие, современный геомаркетинг - многоаспектен. В зависимости от аспекта рассмотрения можно по-разному определить возможности геомаркетинга и его место. Аспект простых приложений (туризм, логистика, размещение товара) позволяет отнести геомаркетинг к новым информационных технологиям, применяемым для решения задач маркетинга и логистики [11].

Аспект интеграции трех групп данных "место", "время", "тема" и выбор в качестве независимой характеристики группу "время" позволяет рассматривать геомаркетинговые исследования как разновидность технологии геомониторинга $[12,13]$. Эта технология включает возможности проведения не только наблюдение и анализа, но и возможности прогнозирования и управления.

Аспект интеграции данных в единую интегрированную информационную систему, привносит в эту систему данных свойства соответствия и эквивалентности между отдельными компонентами, которые имеют разные шкалы оценивания или содержат противоречия. В геомаркетинге появилась возможность на основе использования теории предпочтений обрабатывать эти данные.

Аспект интеграции трех групп данных "место", "время", "тема" и выбор в качестве независимой характеристики группу "место" позволяет использовать геомаркетинг инструмент анализа пространственных процессов, происходящих в современном обществе. Это, например, дает возможность его при отраслевом анализе, например при маркетинге образовательных услуг $[14,15]$. Все это в целом позволяет использовать геомаркетинг как инструмент анализа инвестиционных и инновационных процессов.

Геомаркетинг дает дополнительные возможности пространственного анализа и визуальной обработки информации. Но главным его отличием является возможность проведения комплексного анализа глобальных и локальных процессов. Это делает его важным инструментом для пространственной экономики.

Теория геомаркетинга дает инструмент комплексирования и многоаспектного анализа различных видов информации, различных по масштабу объектов пространственной экономики, различных (глобальных и локальных) рынков в единой информационной среде. 
Технология геомаркетинга как новая интегрированная информационная технология является инструментом анализа экономической информации.

\section{Геомаркетинговые исследования}

Важное значение в геомаркетинге имеют геомаркетинговые исследования. Геомаркетинговые исследования многоаспектны. Они предшествуют анализу эффективности инвестиций в разных областях. Геомаркетинговые исследования являются синтезом геоинформационных исследований, маркетинговых исследований и новых информационных технологий. Они являются развитием маркетинговых исследований на современном уровне информатизации и глобализации общества. Геомаркетинговые исследования представляют собой специальный класс технологий. Их можно разбить на три части: разработка концепций геомаркетинговых исследований, информационное моделирование как метод оптимизации сбора и хранения информации, предметные исследования (Рис. 1)

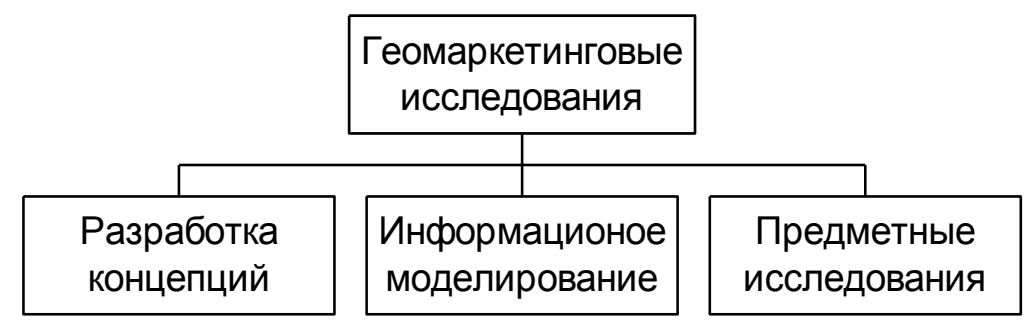

Puc. 1. Основные составляющие геомаркетинговых исследований

При маркетинговых и геомаркетинговых исследованиях большую роль играет выявление тенденций и обнаружение новых качеств. При качественном анализе несущественными оказываются многие количественные характеристики исследуемых процессов. Именно "анализ качеств", а не чисел, стал основным лейтмотивом множества разделов математики, родившихся в XX в. топологии, теории катастроф [16], некоторыХ теорий в нелинейной динамике.

По этой причине при организации данных в геоинформатике и, соответственно, в геомаркетинге важным качеством данных является описание линейных и площадных (ареальных) объектов. Одним из направлений качественного анализа является визуальная обработка информации. Она основана на развитии методов визуализации данных, включающих, как частный случай, картографическое представление информации. Важным является то, что это представление использует динамические модели. Поэтому оперативное введение новой информации приводит к оперативному изменению ее визуального представления.

Пространственный анализ объектов и связей между ними, осуществляемый в технологиях геомаркетинга позволяет решать по новому известные задачи. Это задачи размещения, задачи логистики, задачи управления недвижимостью, операции на земельном рынке.

Ситуационный анализ в геомаркетинге. Новым в теории геомаркетинга является использование специализированных информационных моделей, таких как информационная ситуация, информационная позиция [17] информационное преимущество информационное богатство [18]. Кроме того, ситуационный анализ [19] использует важное свойство пространственных моделей - топологическую структуру. Топологический анализ реальных пространственных объектов является существенным преимуществом геомаркетинга перед другими видами исследований. Этот вид анализа обладает наглядностью и существенно снижает нагрузку на лицо, принимающее решение (ЛПР). Как необходимость снижения информационной нагрузки современному этапу исследований свойственна опора на информационные модели. 
Формирование модели позволяет редуцировать большое количество количественных данных к одному качеству и осуществлять качественный анализ, снижая информационную нагрузку на ЛПР. Это обусловлено тем, что при изучении объектов окружающего мира современными методами результаты исследований можно рассматривать как некие информационные комплексы. Информационные объемы этих комплексов таковы, что исключают возможность непосредственного анализа их человеком.

В качестве первичных средств анализа таких информационных наборов применяют информационные модели, а не непосредственно человеческий интеллект. Этот подход позволяет заменять анализ «количеств» анализом «качеств». Ситуационный анализ редуцирует множество данных к моделям ситуаций и тенденций их изменения. По существу, он заменяет анализ данных на анализ специализированных моделей и на анализ ситуаций, которые описывают эти модели. Этим сокращает объем рутинных работ и повышается качество принимаемых решений.

\section{Выводы}

Информатизация и новая экономика выдвигают новые требования к информационным технологиям и разработке новых инструментов анализа и управления. Одним из таких инструментов анализа и управления в рамках новой экономики [10] является геомаркетинг, как отклик на требования выдвигаемые экономикой. Геомаркетинг использует геоданные, которые являются системным информационным ресурсом [21], что позволяет эффективно применять методы системного анализа в геомаркетинге. Геомаркетинг использует пространственные отношения и гереференции [21], которые позволяют учитывать пространственные факторы и распределение экономических характеристик в реальном пространстве. Геомаркетинг позволяет решать задачи субсидиарного или сетецентрического управления [22]. Геомаркетинг является новым научным направлением в области управления и организации производства и отражает современные микро и макроэкономические тенденции.

\section{Примечания:}

1. Журкин И.Г., Цветков В.Я. Геомаркетинг и ГИС // Информационные технологии. №7. 1998. С. 11-13.

2. Цветков В.Я. Проблемы геомаркетинга // Наука производству. 1998. № 5. С. 2-5.

3. Цветков В.Я. Геомаркетинг. М.: Машиностроение, 2000. 64 с.

4. Цветков В.Я. Задачи геомаркетинга // Геодезия и аэрофотосъемка. 2000. №5. C. $146-154$.

5. Цветков В.Я. Геомаркетинг. М.: Финансы и статистика, 2002. 240 с.

6. Cliquet G. (ed.). Geomarketing: Methods and strategies in spatial marketing. John Wiley \& Sons, 2013. $659 \mathrm{p}$.

7. Schüssler F. Geomarketing: Anwendungen Geographischer Informationssysteme im Einzelhandel. Tectum-Verlag, 2000.

8. Roughgarden J. The theory of coevolution // Coevolution. 1983. C. 33-64.

9. Тихонов А.Н., Иванников А.Д., Соловьёв И.В., Цветков В.Я. Основы управления сложной организационно-технической системой. Информационный аспект. М.: МаксПресс, 2010. 228 c.

10. Tapscott D. The Digital Economy: Promise and Peril in the Age of Networked //Intelligence. 1995. p. 291.

11. Маркелов B.M. Логистика и пространственная экономика / Материалы международного образовательного форума «Бургас 2013». Бургас: Изд-во «ЕООД ИХНИИТ», 2013. С. 91-95

12. Tsvetkov V.Ya. Global Monitoring // European Researcher, 2012, Vol.(33), № 11-1, p. $1843-1851$.

13. Sohn Y. K. et al. Geoheritages and geomonitoring with special references to Jeju Island //Journal of the Geological Society of Korea. 2009. T. 45. C. 751-770.

14. Савиных В.П., Цветков В.Я. Маркетинг образовательных услуг//Известия высших учебных заведений. Геодезия и аэрофотосъемка. 2007. № 4. С. 169-176. 
15. Ожерельева Т.А. Особенности развития маркетинга образовательных услуг// Международный журнал экспериментального образования. 2013. №3. С. 113-115.

16. Zeeman E.C. Catastrophe theory. - Springer Berlin Heidelberg, 1979. p. 12-22.

17. Tsvetkov V.Ya. Information Situation and Information Position as a Management Tool // European Researcher, 2012, Vol.(36), № 12-1, p. 2166-2170.

18. Соловьёв И.В. Информационные ресурсы /научная монография. М.: Московский государственный технический университет радиотехники, электроники и автоматики МГТУ МИРЭА, 2014. 86 c.

19. Цветков В Я., Маркелов В.М. Пространственный ситуационный анализ // Вестник МГТУ МИРЭА. 2013. № 1 (1). С. 103-116.

20. Savinykh V. P., Tsvetkov V. Ya. Geodata As a Systemic Information Resource. Herald of the Russian Academy of Sciences, 2014, Vol. 84, No. 5, pp. 365-368.

21. Майоров А.А., Цветков В.Я. Геореференция как применение пространственных отношений в геоинформатике // Известия высших учебных заведений. Геодезия и аэрофотосъемка, 2012. №3. с. 87-89.

22. Тихонов А.Н., Иванников А.Д., Соловьёв И.В., Цветков В.Я., Кудж С.А. Концепция сетецентрического управления сложной организационно-технической системой. М.: МаксПресс, 2010. 136 с.

\section{References:}

1. Zhurkin I.G., Tsvetkov V.Ya. Geomarketing and GIS // Information Technology. №7. 1998. p. 11-13.

2. Tsvetkov V.Ya. Problems geomarketing // Science production. Number p. 2-5 May 1998.

3. Tsvetkov V.Ya. Geomarketing. M .: Mechanical Engineering, 2000. 64 p.

4. Tsvetkov V.Ya. Tasks geomarketing // Geodesy and aerial photography, 2000, №5, p. 146-154.

5. Tsvetkov V.Ya. Geomarketing- M .: Finance and statistics, 2002. 240 p.

6. Cliquet G. (ed.). Geomarketing: Methods and strategies in spatial marketing. John Wiley \& Sons, 2013. $659 \mathrm{p}$.

7. Schüssler F. Geomarketing: Anwendungen Geographischer Informationssysteme im Einzelhandel. Tectum-Verlag, 2000.

8. Roughgarden J. The theory of coevolution // Coevolution. 1983. p. 33-64

9 Tikhonov A.N., Ivannikov A.D., Soloviev I.V., Tsvetkov V.Ya. Fundamentals of management of complex organizational and technical system. Information aspect. M.: MaksPress, 2010. 228 p.

10. Tapscott D. The Digital Economy: Promise and Peril in the Age of Networked //Intelligence. 1995. p. 291.

11. Markelov V.M. Logistics and spatial economics / Materials of the international educational forum "Burgas 2013." Burgas.: Publishing house "Ltd. IHNIIT" p. 91-95. 2013.

12. Tsvetkov V.Ya. Global Monitoring // European Researcher, 2012, Vol.(33), № 11-1, p. $1843-1851$.

13. Sohn Y. K. et al. Geoheritages and geomonitoring with special references to Jeju Island //Journal of the Geological Society of Korea. 2009. T. 45. p. 751-770.

14. Savinyh V.P., Tsvetkov V.Ya. Marketing of educational services // News of higher educational institutions. Surveying and aerial photography. 2007. № 4. p. 169-176.

15. Ozhereleva T.A. Features Development Marketing of educational services // International Journal of Experimental Education. 2013. №3. p. 113-115.

16. Zeeman E. C. Catastrophe theory. - Springer Berlin Heidelberg, 1979. p. 12-22.

17. Tsvetkov V. Ya. Information Situation and Information Position as a Management Tool // European Researcher, 2012, Vol.(36), № 12-1, p. 2166-2170.

18. Soloviev I.V. Information resources / monograph. M.: Moscow State Technical University of Radio Engineering, Electronics and Automation Bauman MIREA, 2014. 86p.

19. Tsvetkov V.Ya., Markelov V.M. Spatial situational analysis // MSTU MIREA HERALD. 2013. № 1 (1). p. 103-116.

20. Savinykh V. P., Tsvetkov V. Ya. Geodata As a Systemic Information Resource. Herald of the Russian Academy of Sciences, 2014, Vol. 84, No. 5, pp. 365-368 
21. Mayorov A.A., Tsvetkov V.Ya. Georeference of how the use of spatial relations in geoinformatics // News of higher educational institutions. Surveying and aerial photography, 2012. №3. P. $87-89$.

22. Tikhonov A.N., Ivannikov A.D., Soloviev IV, Tsvetkov V.Ya., Kudza S.A. The concept of network-centric management of complex organizational and technical systems. M: MaksPress, 2010. 136 p.

УДК 339.138

\section{Развитие геомаркетинга}

Татьяна Алексеевна Ожерельева

Московский государственный университет геодезии и картографии Москва, Российская Федерация

Старший преподаватель

E-mail: ozerotana@yandex.ru

Аннотация. Статья анализирует состояние и развитие геомаркетинга. Статья показывает многоаспектность геомаркетинга: прикладная технология, технология управления. Показано что геомаркетинг можно рассматривать как отражение процессов коэволюции в обществе. Раскрываются особенности геомаркетинговых исследований и ситуационного анализа в геомаркетинге. Описаны приложения геомаркетинга.

Ключевые слова: маркетинг; геомаркетинг; пространственная экономика; информационные технологии; информационные технологии управления. 